\title{
Regression of Sebaceous Carcinoma of the Eyelid after a Small Incisional Biopsy: Report of Two Cases
}

\author{
Seymour Brownstein ${ }^{a, b}$ Sonul Mehtac Navdeep Nijhawan ${ }^{c}$ Guy Allaire ${ }^{d}$ \\ Tuan Quynh Tram Nguyen ${ }^{\mathrm{e}}$ Tina Tang $^{\mathrm{a}, \mathrm{b}}$ Kailun Jiang $^{\mathrm{a}, \mathrm{b}}$ Solin Saleh ${ }^{\mathrm{a}, \mathrm{b}}$ \\ Evan Kalin-Hajdu $^{\mathrm{e}}$ Robbin Bruen ${ }^{\mathrm{e}}$ James Oestrelcher ${ }^{\mathrm{C}}$ \\ ${ }^{a}$ Department of Ophthalmology, The Ottawa Hospital and University of Ottawa, Ottawa, ON, Canada; ${ }^{b}$ Department \\ Pathology, The Ottawa Hospital and University of Ottawa, Ottawa, ON, Canada; ' Department of Ophthalmology, \\ University of Toronto, Toronto, ON, Canada; ${ }^{\mathrm{d}}$ Department of Pathology, University of Montreal, Montreal, QC,

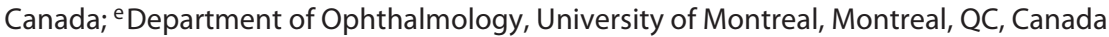

\section{Established Facts}

- Regression after incisional biopsy of cutaneous basal cell carcinoma, squamous cell carcinoma, and Merkel cell carcinoma of the eyelid has been reported. Spontaneous regression of retinoblastoma is a well-established finding, while spontaneous regression of choroidal melanoma has been documented in rare cases.

\section{Novel Insights}

- To the best of our knowledge, we report for the first time 2 cases of sebaceous carcinoma of the eyelid with total long-term clinical regression of the tumor following incisional biopsy.

\section{Keywords}

Sebaceous carcinoma · Ptosis · Eyelid · Orbit · Spontaneous regression · Exenteration $\cdot$ Small incisional biopsy

\section{Abstract \\ Purpose: To report 2 cases of regression of sebaceous carci- noma of the eyelid after a small incisional biopsy. Methods: Clinical, imaging, and histopathological findings are pre- sented, with a literature review on regressing ocular tumors. Results: Our first patient was a 79-year-old man who pre-}

(c) 2018 S. Karger AG, Basel sented with a 10-month history of progressive left upper eyelid ptosis caused by an eyelid tumor with orbital involvement and confirmed on magnetic resonance imaging. Our second patient was a 70-year-old woman who presented with ptosis with a left upper eyelid mass. Both patients underwent a small incisional biopsy of their lesion. The histopathological diagnoses in both cases were consistent with sebaceous carcinoma. Both patients refused exenteration.

Presented in part at the June 2015 International Congress of Ocular Oncology, Paris, France.
Seymour Brownstein, MD

The Ottawa Hospital

501 Smyth Road, Suite W6213

Ottawa, ON K1H 8L6 (Canada)

E-Mail sbrownstein@ ohri.ca 
Follow-up clinical examination and imaging disclosed total regression of the ptosis and of the neoplasm with no sign of recurrence in both patients over a 4-year period for Case 1 and a 7-year period for Case 2. Conclusion: Regression following incisional biopsy of basal cell, squamous cell, and Merkel cell carcinoma, including of the eyelid, is well documented. To the best of our knowledge, our 2 cases of sebaceous carcinoma are the first to be reported with total involution clinically and on imaging of the tumor following partial incisional biopsy.

(c) 2018 S. Karger AG, Basel

\section{Introduction}

Regression after incisional biopsy of cutaneous basal cell carcinoma (BCC) and squamous cell carcinoma (SCC) is a well-known phenomenon [1]. This has also been reported for Merkel cell carcinoma [2]. Cases of spontaneous regression without surgical intervention have been reported in retinoblastoma [3] and choroidal melanoma [4]. The underlying mechanisms for this phenomenon are currently being studied [1-7]. We report, to the best of our knowledge, the first 2 cases in the current literature of regressed sebaceous carcinoma (SebCC) after a small incisional biopsy.

\section{Clinical History}

Case 1

A 79-year-old man was referred with a 10-month history of progressive left upper eyelid ptosis, beginning 4 months previously as a "bulge" under the eyelid.

On presentation, the visual acuity was $20 / 30$ for the right eye (OD) and 20/40 for the left eye (OS). The intraocular pressure was $14 \mathrm{~mm} \mathrm{Hg}$ OD and $19 \mathrm{~mm} \mathrm{Hg}$ OS. On external examination, he had $5 \mathrm{~mm}$ of ptosis and a firm mass in the left upper and lower eyelids (Fig. 1a). Hertel exophthalmometry disclosed no proptosis. The extraocular movements of his left eye were restricted when looking superiorly and laterally. The anterior segment and dilated funduscopic examination were unremarkable except for mild nuclear sclerotic cataracts.

Magnetic resonance imaging of the orbits revealed a soft tissue mass involving the left eyelids and orbit (Fig. 1b). The major bulk of the mass was located in the superior aspect of the left medial orbit and measured $2.7 \times 2.8 \mathrm{~cm}$ in transverse diameter and $1.4 \mathrm{~cm}$ in thickness medially where it extended toward the anterior ethmoid and the nasal bone (Fig. 1b). Although it did not invade the globe, the mass abutted the anterior aspect of the left medial rectus muscle.

A left anterior orbitotomy with a small upper eyelid crease incision was performed and a specimen measuring $2 \times 2 \mathrm{~mm}$ was excised from the anterior palpable lesion.

Regression of Sebaceous Carcinoma of the Eyelid
Histopathological and immunohistopathological examinations were most consistent with a diagnosis of SebCC (Fig. 2, Case $1)$. Based on these findings, an orbital exenteration was recommended.

Upon follow-up examination 2 weeks after the biopsy, there was gradual improvement in the ptosis and fullness of the orbit.

The patient refused further surgical intervention. He attributed his improvement to an antibiotic, cephalosporin, that his primary care physician gave him for an ear infection during the time period between the biopsy and the follow-up appointment. It was decided to continue treating the patient with oral ciprofloxacin followed by prednisone $(1 \mathrm{mg} / \mathrm{kg} /$ day $)$ and tapered over several weeks. The patient was subsequently followed up with repeat imaging and clinical examinations during the next 4 years and showed complete regression of the tumor and resolution of all symptoms (Fig. 1c, d). We deferred further surgical management and he passed away 1 year later due to cardiac disease unrelated to the prior ocular pathology.

\section{Case 2}

A 70-year old woman presented with a mass in the left upper eyelid, which was diagnosed initially as a chalazion clinically (Fig. 1e, f). The lesion was first evident 3 months previously and slowly increased in size. She had no previous ophthalmic history or surgeries.

The visual acuity was 20/40 OD and 20/80 OS. Slit-lamp and funduscopic examinations were otherwise within normal limits except for bilateral nuclear sclerotic cataracts. Her external ocular examination showed a $3-\mathrm{cm}$ firm hyperemic tarsal mass extending from the left medial canthal area to the central tarsus of the left upper eyelid with adjacent madarosis (Fig. 1e, f).

Computed tomographic (CT) imaging showed an enhancing nodular, multi-loculated lesion in the medial left upper eyelid, measuring $3 \mathrm{~cm}$ transversely and $1.5 \mathrm{~cm}$ in axial depth. Nasally, it was indistinguishable from the insertion of the medial rectus muscle and sclera with deformation of the globe. Temporally, it also involved the insertions of the superior oblique and lateral rectus muscles. Two biopsy specimens of the lesion, measuring $10 \times$ $4 \mathrm{~mm}$, were obtained and were consistent with a diagnosis of SebCC.

The patient refused further treatment including exenteration. She was lost to follow-up until 24 months after initial presentation when, with no therapeutic intervention, there was involution of the left eyelid mass clinically and on CT imaging (Fig. 1h-j). The patient's most recent examination, 7 years after initial presentation, disclosed no evidence of recurrent tumor or regional metastatic disease.

\section{Histopathological Findings}

Representative histochemical and immunohistopathological findings utilizing comparative stains are listed side by side in Figure 2 with Case 1 on the left and Case 2 on the right.

The histopathological findings of both cases were mostly similar. Each consisted of fragments of tissue, which were infiltrated by neoplastic lobules of epithelial 

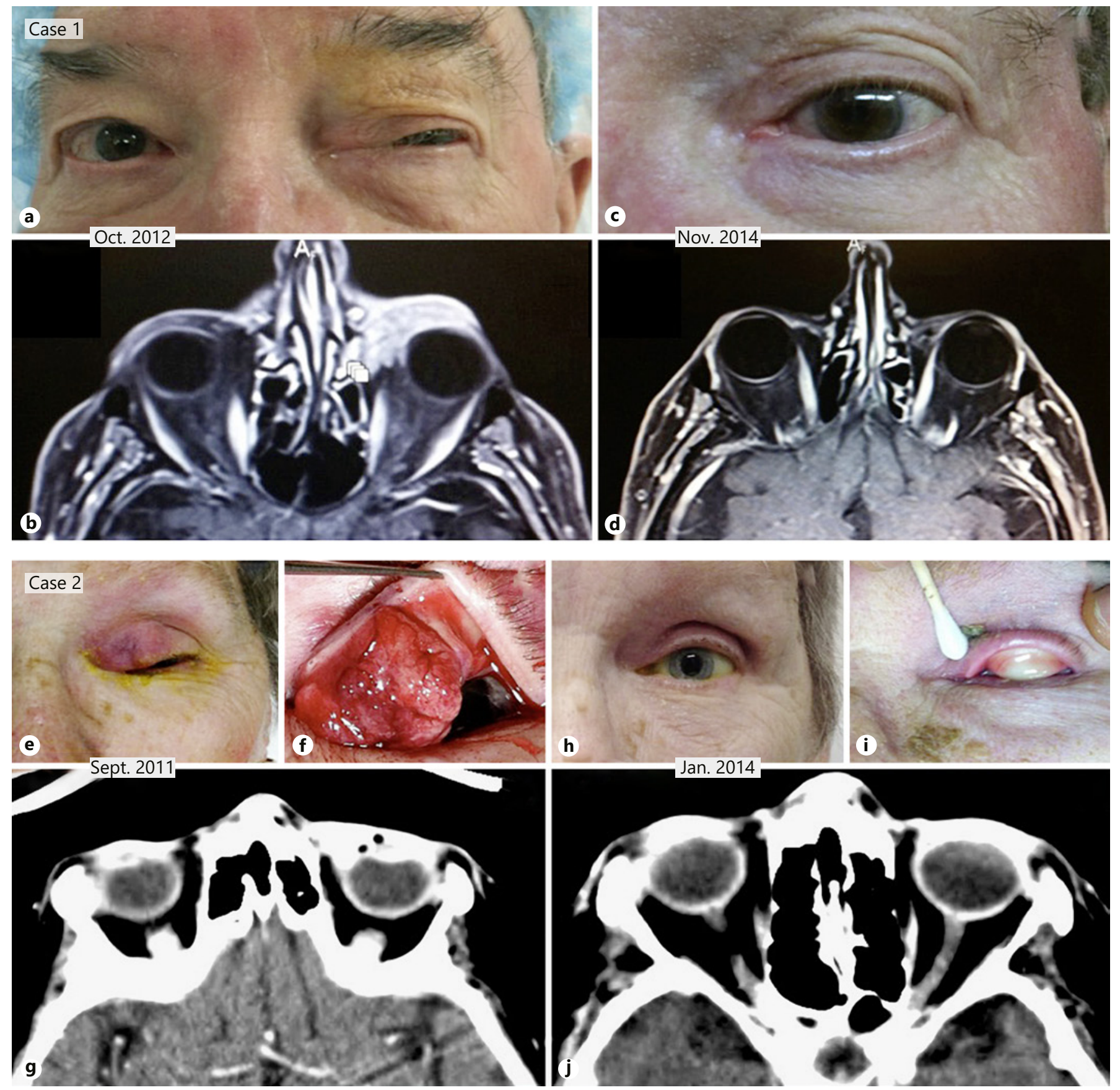

Fig. 1. a Our first case (Case 1) demonstrates marked ptosis and substantial fullness in the medial upper and lower eyelids of the left eye. b Magnetic resonance imaging (MRI) at initial presentation revealed a soft tissue mass involving the left eyelids, especially medially extending into the left anterior medial orbit. c 14 months after incisional biopsy of the medial eyelid with orbital extension, Case 1 shows complete clinical resolution of the lesions. d MRI 14 months after the small incisional biopsy shows no evidence of the prior tumor, which has undergone regression. e Case 2 has a large

mass in the medial left upper eyelid, which shows ptosis. $f$ Eversion of the eyelid reveals a massive lesion in the anterior medial eyelid. g Computed tomographic (CT) imaging of Case 2 exhibits a contrast-enhancing loculated lesion of the medial left upper eyelid. $\mathbf{h}, \mathbf{i}$ There is complete resolution of the ptosis (h) and complete resolution of the tarsal lesion clinically (i) at 2.5 years after initial biopsy. j Follow-up CT scan 2.5 years after initial presentation discloses regression of the tumor. 
cells of variable size and shape involving the tissue margins in many areas (Fig. 2a, b). The atypical epithelial cells showed pleomorphic and vesicular nuclei, with prominent and multiple nucleoli, and a foamy cytoplasm focally, more so in Case 2. Both cases showed inflammatory infiltrates adjacent to and within the neoplasm, which

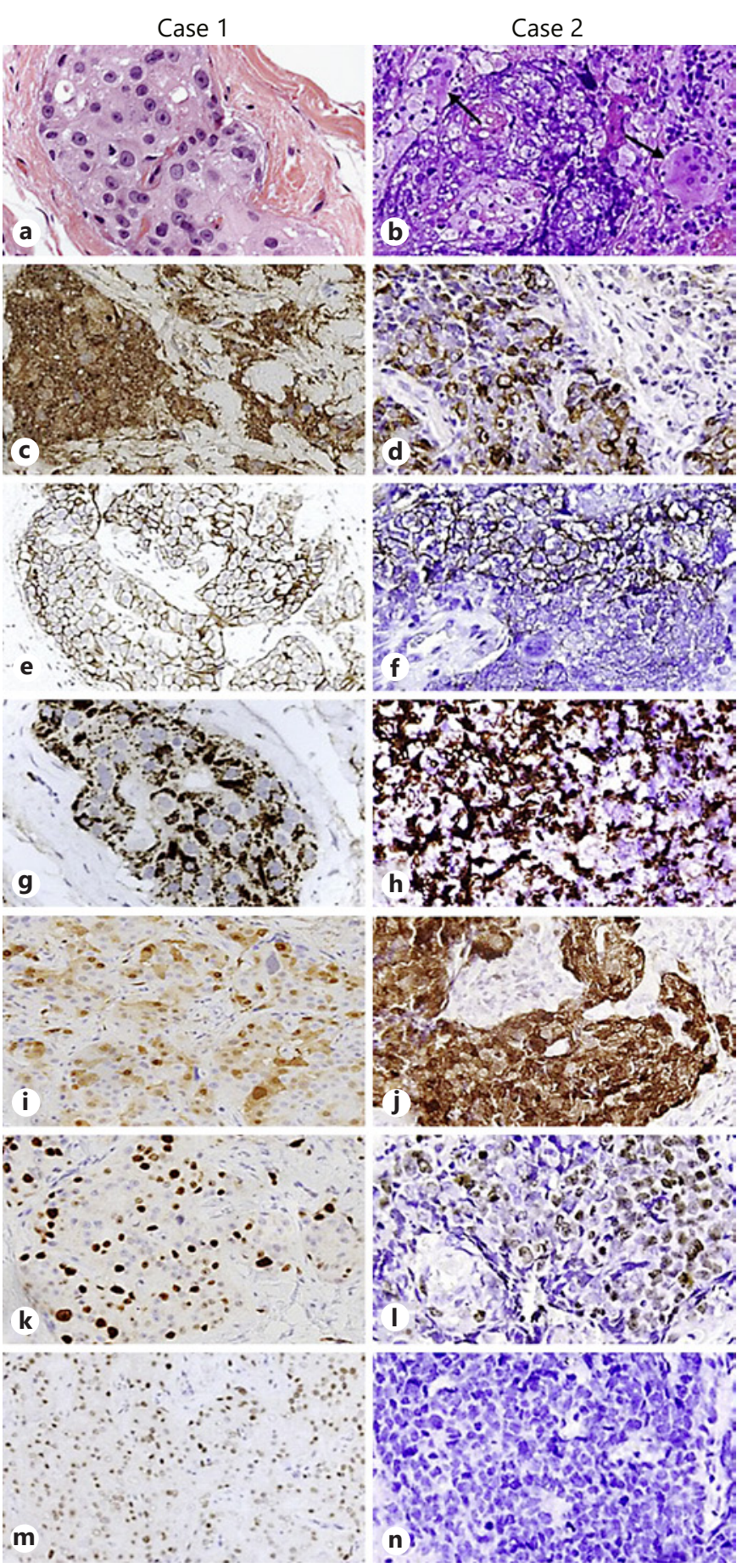

Regression of Sebaceous Carcinoma of the Eyelid were more extensive in Case 2 where there also were focal chronic granulomatous inflammatory infiltrates. In contrast, Case 1 showed mostly a mild to moderate nongranulomatous inflammatory infiltrate.

The atypical cells stained positive for epithelial membrane antigen (EMA) (Fig. 2c, d), BER-EP4, more so in Case 1 (Fig. 2e, f), adipophilin (Fig. 2g, h), and P16 (Fig. 2i, j). Ki67 stained 35\% of the neoplastic cells' nuclei in Case 1 and $60 \%$ in Case 2 (Fig. $2 \mathrm{k}, \mathrm{l}$ ). Androgen receptor stained strongly positive in Case 1 and negative in Case 2 (Fig. $2 \mathrm{~m}, \mathrm{n}$ ).

The findings were most consistent with a diagnosis of SebCC in both cases, which clinically underwent regression on long-term follow-up at 4 and 7 years, respectively.

\section{Discussion}

Regression following small incisional biopsy of cutaneous cancer is well known to occur in BCC and SCC [1]. Swetter et al. [1] studied regression of nonmelanoma skin cancer after incisional biopsy. Her team studied 910 transections of basal cell and SCC and found a regression rate of $24 \%$ with a follow-up of 6.5 years [1]. This study also showed that SCC (40\%) is more likely to regress than BCC (20\%) [1]. Regression after incisional biopsy has also been reported to occur in Merkel cell carcinoma of the head and neck, including the eyelid [2]. Furthermore, spontaneous regression of retinoblastoma has been well documented with reports at rates of 2\% [3], and rarely in cases of choroidal melanoma [4]. To the best of our knowledge, regression has never been reported to occur in SebCC.

From our literature review, we found only 1 previously reported patient's sebaceous lesion sharing similar clinical and histopathological features with our Case 2

Fig. 2. The findings in Case 1 are in the left column, while those from identical stains are shown in the right column side by side. a Biopsy specimen from Case 1 is comprised of glandular tissue with pleomorphic nuclei and focal foamy cytoplasm consistent with the findings in a sebaceous adenocarcinoma. Hematoxylineosin, original magnification $\times 400$. $\mathbf{b}$ Biopsy specimen from Case 2 shows a lesion that contained cells with pleomorphic nuclei and foamy cytoplasm with focal chronic granulomatous inflammatory infiltrates (arrows). Hematoxylin-eosin, original magnification $\times 200$. c, d Epithelial membrane antigen is strongly positive in the tumor cells. $\times 100$. e, $\mathbf{f}$ BER-EP4 is positive, more so in Case $1 . \times 100$. $\mathbf{g}, \mathbf{h}$ The tumor cells are immunoreactive for adipophilin in both cases. $\times 100$. i, j P16 is highly positive in both cases. $\times 100$. k, I Ki67 is positive in $35 \%$ of the tumor cells in Case 1 and in $60 \%$ in Case 2. $\times 100$. $\mathbf{m}, \mathbf{n}$ Staining for androgen receptor is very positive in Case 1 but negative in Case 2. $\times 100$.
Ocul Oncol Pathol 2019;5:252-257

DOI: $10.1159 / 000490706$ 
[8]. Hwang et al. [8] reported a patient presenting with a yellowish lesion protruding from the posterior lamella of the left upper eyelid. Histopathologically, there were small foci of SebCC, but the bulk of the lesion, including the margins, was composed of a dense collection of foamy histiocytes and multinucleated giant cells [8]. The granulomatous reaction, similar to that in our Case 2, may indicate a spontaneous local inflammatory reaction to the tumor that was further augmented by the wound-healing process following the biopsy, with both processes contributing to the tumor regression [1].

Sebaceous gland carcinoma is a highly malignant and potentially lethal tumor with a reported mortality rate of about 6\% [9]. Periocular SebCC can arise from the meibomian glands of the tarsal plate, from the eyelid glands of Zeis, or from the sebaceous glands of the caruncle, eyebrow, or facial skin [9]. In SebCC, there is a tendency for the tumor within the tarsal plate to progress to an intraepithelial growth phase [9]. Orbital exenteration or wide surgical excision is often necessary for the management of SebCC that is widespread and aggressive [9]. The 2 patients in our report both refused exenteration, but their follow-up clinical examinations and radiological imaging showed complete resolution of this tumor and no signs of local recurrence over a 4-year period for Case 1 and a 7 -year period for Case 2 .

SebCC is characteristically an unencapsulated infiltrating mass with cells composed of finely vacuolated, frothy cytoplasm [9]. Mitotic activity can be high with nuclear pleomorphism [9]. In order to improve the accuracy in the diagnosis of SebCC, several publications report various immunohistochemical stains that may be useful in differentiating SebCC from BCC and SCC [911]. Mulay et al. [10] reported EMA positivity in $100 \%$ of SCC and 96\% of SebCC, while BCC was negative. Jakobiec and Mendoza [11] reported EMA positively and Ki67 with an average $45 \%$ proliferation index (range 27.465.8\%) in eyelid SebCC. Bell et al. [12] reported p16, a tumor suppressor, positivity in $96 \%$ of SebCC. Several reports have focused on the usefulness of adipophilin immunostaining in the diagnosis of sebaceous lesions [11, $13,14]$. Adipophilin is expressed in a membranous and vesicular pattern in sebaceous tumors, while a nonspecific granular pattern of staining is found in other tumors such as BCC and SCC [13]. Variable results have been reported for BER-EP4 and androgen receptor staining in SebCC $[10,11,15]$. The histopathological features and overall immunohistochemical analysis of both of our cases, as demonstrated in Table 1 and Figure 2, were consistent with a diagnosis of SebCC [9-15].
Table 1. Immunohistochemical profile of biopsy specimens

\begin{tabular}{lll}
\hline Immunohistochemical stains & Case 1 & Case 2 \\
\hline EMA & $4+$ & $4+$ \\
BER-EP4 & $4+$ & $2+$ \\
Adipophilin & $3+$ & $4+$ \\
P16 & $4+$ & $4+$ \\
Ki67 & $2+(35 \%)$ & $3+(60 \%)$ \\
Androgen receptor & $4+$ & $0 \%$ \\
\hline
\end{tabular}

Although extremely rare, our 2 cases document that regression of a highly malignant tumor, such as SebCC, is possible. While the exact mechanism of tumor regression remains to be elucidated, we hypothesize that biopsy and manipulation of the tumor triggered an immune-mediated cascade of regression as suggested in cases of regressed SCC and BCC [1]. In Case 1, cefprozil, a cephalosporin antibiotic, was given following the biopsy and prior to the follow-up appointment that was given by the family physician for an ear infection. Bessler et al. [16] looked at the effect of cefotaxime, also a cephalosporin antibiotic, in causing a substantial enhancement of IL-2 production, which has been found to be increased in regressed BCC [7]. Case 2 highlights that SebCC regression can also occur without introduction of other pharmacological agents and may be associated with the incisional biopsy augmenting a local immune response with extensive granulomatous inflammation that may lead to a regression of the tumor [1].

\section{Conclusion}

Most cases of incompletely excised SebCC have a poor outcome, while in our 2 cases, for reasons that have not been well established, their tumors underwent total clinical regression [9]. Further research into the mechanisms of this phenomenon should be pursued.

\section{Statement of Ethics}

The patients and/or close relatives have given informed consent for the publication of the clinical photographs and the medical findings. The institution review board of the Ottawa Hospital waived ethics review because this was a case report that conformed to the Code of Ethics of the World Medical Association (Declaration of Helsinki) and obtained informed patient consent. 


\section{Disclosure Statement}

The authors have no financial conflict of interest to disclose. All of the authors have seen and given their approval for the submis- sion of this paper. They warrant that this article is the authors' original work, has not received prior publication, and is not under consideration for publication elsewhere.

\section{References}

1 Swetter SM, Boldrick JC, Pierre P, Wong P, Egbert BM. Effects of biopsy-induced wound healing on residual basal cell and squamous cell carcinomas: rate of tumor regression in excisional specimens. J Cutan Pathol. 2003 Feb;30(2):139-46.

2 Missotten GS, de Wolff-Rouendaal D, de Keizer RJ. Merkel cell carcinoma of the eyelid review of the literature and report of patients with Merkel cell carcinoma showing spontaneous regression. Ophthalmology. 2008 Jan; 115(1):195-201.

3 Sanborn GE, Augsburger JJ, Shields JA. Spontaneous regression of bilateral retinoblastoma. Br J Ophthalmol. 1982 Nov;66(11):68590.

4 Nathanson. Spontaneous regression of malignant melanoma: a review of the literature on incidence, clinical features, and possible mechanisms. Natl Cancer Inst Monogr. 1976 Nov;44:67-76.

5 Claudy AL, Viac J, Schmitt D, Alario A, Thivolet J. Identification of mononuclear cells infiltrating basal cell carcinomas. Acta Derm Venereol. 1976;56(5):361-5.
6 Viac J, Bustamante R, Thivolet J. Characterization of mononuclear cells in the inflammatory infiltrates of cutaneous tumours. Br J Dermatol. 1977 Jul;97(1):1-10.

7 Hunt MJ, Halliday GM, Weedon D, Cooke $\mathrm{BE}$, Barnetson RS. Regression in basal cell carcinoma: an immunohistochemical analysis. Br J Dermatol. 1994 Jan;130(1):1-8.

8 Hwang FS, Neekhra A, Lucarelli MJ, Warner TF, Snow SN, Albert DM. Sebaceous cell carcinoma of the eyelid: a rapidly enlarging lesion with massive xanthogranulomatous inflammation. Ophthal Plast Reconstr Surg. 2010 May-Jun;26(3):208-10.

9 Shields JA, Demirci H, Marr BP, Eagle RC Jr, Shields CL. Sebaceous carcinoma of the ocular region: a review. Surv Ophthalmol. 2005 Mar-Apr;50(2):103-22.

10 Mulay K, White VA, Shah SJ, Honavar SG. Sebaceous carcinoma: clinicopathologic features and diagnostic role of immunohistochemistry (including androgen receptor). Can J Ophthalmol. 2014 Aug;49(4):326-32.
11 Jakobiec FA, Mendoza PR. Eyelid sebaceous carcinoma: clinicopathologic and multiparametric immunohistochemical analysis that includes adipophilin. Am J Ophthalmol. 2014 Jan;157(1):186-208.e2.

12 Bell WR, Singh K, Rajan Kd A, Eberhart CG. Expression of p16 and p53 in intraepithelial periocular sebaceous carcinoma. Ocul Oncol Pathol. 2015 Dec;2(2):71-5.

13 Ostler DA, Prieto VG, Reed JA, Deavers MT, Lazar AJ, Ivan D. Adipophilin expression in sebaceous tumors and other cutaneous lesions with clear cell histology: an immunohistochemical study of 117 cases. Mod Pathol. 2010 Apr;23(4):567-73.

14 Milman T, Schear MJ, Eagle RC Jr. Diagnostic utility of adipophilin immunostain in periocular carcinomas. Ophthalmology. 2014 Apr; 121(4):964-71.

15 Sramek B, Lisle A, Loy T. Immunohistochemistry in ocular carcinomas. J Cutan Pathol. 2008 Jul;35(7):641-6.

16 Bessler H, Gurary N, Aloni D, Vishne TH, Sirota L. Effect of cefotaxime on cytokine production in newborns and adults in vitro. Biomed Pharmacother. 2000 Aug; 54(7): $410-4$. 